\title{
Una aproximación a la realidad aumentada y sus aplicaciones quirúrgicas $^{1}$
}

\section{An approach to augmented reality and the surgical applications}

\author{
Uma abordagem ao aumento da realidade e suas aplicações \\ cirúrgicas
}

\author{
E. Aguilar, A. Vivas, y J. Sabater
}

Recibido: diciembre 13 de 2016 - Aceptado: junio 30 de 2018

\begin{abstract}
Resumen- Este artículo muestra un panorama general de las temáticas de investigación y desarrollo en el área de la simulación en el entrenamiento y apoyo de cirugías usando la tecnología de Realidad Aumentada, la cual se caracteriza por enriquecer la percepción sensorial adicionando contenido virtual sobre la percepción de realidad. Inicialmente, se describen los sistemas de guía de ayuda para cirugía, luego, los principales avances en simuladores usando como base de comparación los simuladores de Realidad Virtual (VR) y, para finalizar, se realiza una exposición de aplicaciones de Realidad Aumentada (AR) en la cirugía haciendo énfasis en las cirugías mínimamente invasivas. En esta revisión se muestra el rol de la simulación en el entrenamiento quirúrgico y en la cirugía guiada, así como la ventaja comparativa de los entrenadores de Realidad Aumentada (AR) sobre los de Realidad Virtual (VR).

Palabras clave - cirugía laparoscópica, realidad aumentada, realidad virtual, simulación quirúrgica.
\end{abstract}

${ }^{1}$ Producto derivado del proyecto de investigación "Contribución a la Cirugía Mínimamente Invasiva con Realidad Aumentada", presentado como proyecto doctoral de la estudiante Eliana Aguilar al programa de Doctorado en Ciencias de la Electrónica de la Universidad del Cauca.

E. Aguilar, Universidad del Cauca, Popayán, Colombia, email: eaguilar@unicauca.edu.co.

A. Vivas, Universidad del Cauca, Popayán, email: avivas@unicauca. edu.co.

J. Sabater, Universidad Miguel Hernández de Elche en España, email: j.sabater@goumh.umh.es.

Como citar este artículo: Aguilar, E., Vivas, A. y Sabater, J. Una Aproximación a la Realidad Aumentada y sus Aplicaciones Quirúrgicas, Entre Ciencia e Ingeniería, vol. 12, no. 24, pp.15-24, julio-diciembre, 2018. DOI: http://dx.doi.org/10.31908/19098367.3811

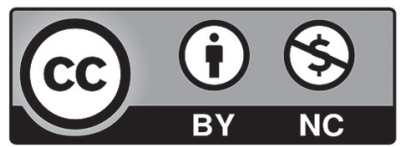

Attribution-NonCommercial 4.0 International (CC BY-NC 4.0)
Abstract - This paper shows an overview of simulation in training and support of surgeries with Augmented Reality, AR is a technology characterized by sensor perception enrichment adding virtual content to the perception of reality. At first this article shows an overview of guided surgery systems and the principal advances in simulation using Virtual Reality (VR), and finally, it presents a group of Augmented Reality (AR) applications with emphasis on minimally invasive surgeries. In this review, it is concluded that one of the comparative advantages of the training Augmented Reality (AR) with Virtual Reality (VR) is the haptic feedback. The new researches in this area are the visual and haptic realism and dynamic tracking of markers and organs that allows sensory augmentation with high reality affinity.

Keywords - augmented reality, laparoscopic surgery, simulation virtual.

Resumo-Este artigo mostra uma visão geral dos tópicos de pesquisa e desenvolvimento na área de simulação no treinamento e suporte de cirurgias usando a tecnologia Realidade Aumentada, que é caracterizada pela percepção sensorial enriquecedora pela adição de conteúdo virtual sobre a percepção da realidade. Neste artigo, inicialmente são nomeados os sistemas de guia de ajuda para cirurgia, seguidos pelos principais avanços nos simuladores, utilizando os simuladores de Realidade Virtual (VR) como base de comparação e, finalmente, uma exposição de aplicações de Realidade Aumentada (AR) enfatizando cirurgias minimamente invasivas. Nesta revisão, mostramos o papel da simulação no treinamento cirúrgico e cirurgia guiada, bem como a vantagem comparativa de treinadores de Realidade Aumentada (AR) sobre treinadores de Realidade Virtual (RV).

Palavras chave - cirurgia laparoscópica, aumentada, realidade virtual, simulação cirúrgica.

\section{INTRODUCCIÓN}

$\mathrm{D}$ ESDE hace algunos años se ha implementado con éxito un tipo especial de cirugía conocida como 
Cirugía Mínimamente Invasiva (MIS); esta consiste en realizar pequeñas incisiones a través de las que se introduce el instrumental quirúrgico manipulado por el cirujano, incluyendo una cámara para visualizar la zona a ser intervenida [1]. Este tipo de intervención permite la minimización del tamaño y número de orificios de inserción $\mathrm{y}$, por ende, la miniaturización del instrumental usado, lo cual conlleva a la disminución de los cuidados postoperatorios y de hospitalización [2], y a una reducción de los riesgos de complicaciones quirúrgicas para el paciente [3].

La cirugía mínimamente invasiva sin duda trae muchos y muy importantes beneficios para el paciente, pero a su vez involucra un mayor reto para los cirujanos debido a que la miniaturización de las inserciones limita su percepción visual y táctil [4]. El uso del instrumental anula en gran medida la percepción de tacto y es muy difícil para un cirujano determinar si es un tejido duro, suave o elástico, al mismo tiempo que se reduce el campo visual del experto.

De otro lado, el entrenamiento basado en simulación ha sido un área creciente en el proceso de mejora de las competencias de los cirujanos en el campo de las cirugías mínimamente invasivas. Aunque el entrenamiento quirúrgico se realiza desde hace muchos años sobre cadáveres y modelos artificiales, es tan solo hasta hace una década que los simuladores basados en software han comenzado a penetrar con fuerza en los esquemas de entrenamiento quirúrgico, en especial con el uso de simuladores apoyados principalmente en realidad virtual, los cuales se proyectan como una buena alternativa en el entrenamiento en MIS [5] [6].

Es importante destacar que el entrenamiento con realidad virtual permite la flexibilidad en la selección de escenarios de medición de la dinámica de los instrumentos y determinación y seguimiento de errores, posibilitando una automatización del proceso de entrenamiento. Sin embargo, la literatura reconoce como obstáculo para el uso de la realidad virtual, la pobre representación de órganos y escenarios pese a los grandes esfuerzos de realismo en los entornos tridimensionales.

Es por tal razón que el interés en el desarrollo de simuladores virtuales se redirige actualmente hacia otro tipo de tecnología, la cual permite mezclar escenarios reales con objetos virtuales, denominada realidad aumentada. Existe otro término, la virtualidad aumentada, que se caracteriza por desplegar una representación virtual tridimensional usando datos médicos reales y en tiempo real, es decir, la virtualidad se basa en datos reales del escenario o del paciente, en tanto que la realidad aumentada se caracteriza por ser una tecnología que sobrepone imágenes en tiempo real sobre video o fotografías, lo cual puede verse representado en imágenes reales enriquecidas con información necesaria para cumplir un objetivo específico.

Básicamente la realidad aumentada (AR de sus siglas en inglés - augmented reality) tiene como finalidad sobreponer imágenes tridimensionales sobre las imágenes proporcionadas por la cámara [7]. Esta técnica está siendo apropiada en aplicaciones médicas debido principalmente a que genera un incremento de las señales visuales percibidas por el cirujano con una ampliación de su percepción visual, hecho que se ve reflejado en mayor precisión y éxito en las intervenciones; además, mejora la respuesta ojo mano del cirujano gracias a la ampliación de la realidad. En contraposición a la realidad virtual que recrea un ambiente en su mayoría artificial, la realidad aumentada enriquece la realidad, lo que resulta ser de mayor aceptación por el ojo y cerebro humano [8].

Los sistemas anteriormente mencionados ayudan en gran medida a compensar la pérdida de percepción visual y táctil en las cirugías mínimamente invasivas, mientras que la mejoría en aspectos motrices se consigue con sistemas asistidos con robots, obteniéndose resultados satisfactorios en muchas de las actividades quirúrgicas [9].

En este artículo se realiza una exposición del impacto de la realidad aumentada en cirugías mínimamente invasivas. La discusión se realiza con base en los principales avances alcanzados hoy en día en realidad aumentada en la cirugía, las principales tendencias en esta tecnología y la manera como la realidad aumentada contribuye al entrenamiento quirúrgico.

\section{Cirugía Mínimamente INVASIVA}

Con la introducción hace más de 30 años de la cirugía mínimamente invasiva asistida por video, se dio la bienvenida a una gran revolución quirúrgica, rompiendo con el concepto de intrusión producida por las amplias heridas que debían realizarse en las operaciones abdominales [6]. Los procedimientos quirúrgicos mínimamente invasivos han influido en la cirugía moderna por la disminución de la invasividad, minimizando principalmente el tiempo de recuperación del paciente y los costos de hospitalización. Sin embargo, los procedimientos quirúrgicos MIS, al utilizar instrumental de gran longitud insertado a través de pequeños orificios en el cuerpo, privan a los cirujanos de la percepción de profundidad, limitan la destreza y el sentido del tacto, alterando la coordinación mano ojo.

Existen muchas acciones de gran simplicidad en el caso de los procedimientos abiertos (cirugías abiertas), donde los cirujanos dependen de la combinación de su tacto y de sus capacidades visuales para diagnosticar el tejido normal o anormal, lo cual no ocurre en el caso de los procedimientos MIS, puesto que la intervención se realiza de manera indirecta [1]. Las cirugías mínimamente invasivas pueden realizarse en diversas partes del cuerpo, pero cuando la cirugía MIS se realiza en la zona abdominal, se conoce como laparoscopia y puede involucrar diversos tipos de procedimientos en el abdomen como cirugías urológicas, ginecológicas, oncológicas, o en órganos específicos como el páncreas y el hígado.

\section{A. Laparoscopia}

Este procedimiento se basa en el uso de instrumentos quirúrgicos especiales y un sistema de visión con una fuente de luz fría (endoscopio); todos los instrumentos son introducidos a través de pequeñas incisiones en la cavidad abdominal. La imagen de video se transmite por el endoscopio y la cámara endoscópica la opera manualmente un asistente humano, imagen que a su vez es observada en un 
monitor mientras el cirujano manipula los dos instrumentos, guiado por la imagen que se observa; todo esto sin necesidad de realizar una gran abertura del abdomen [10]. La cirugía laparoscópica tiene su mayor grado de exigencia en el uso del instrumental y en la visualización de la profundidad de campo [11].

En la actualidad, las operaciones laparoscópicas son muy utilizadas en todo el mundo, y han dado lugar a otros avances de la cirugía mínimamente invasiva, como NOTES y LESS. NOTES (Natural Orifice Transluminal Endoscopic Surgery), consiste básicamente en un procedimiento quirúrgico realizado a través de orificios naturales y puede ser transoral, transvaginal o transanal [12] [13]. LESS (Laparo Endoscopic Single-Site Surgery) es una técnica usada para varios procedimientos MIS, en la cual solo se deja una cicatriz en el abdomen ya que todo se realiza por un solo orificio [14] [15].

Con la adopción y amplia extensión de la cirugía mínimamente invasiva y sus retos en la precisión de la cirugía y en el dominio del instrumental, programas académicos de medicina y universidades reconocen la necesidad de implementar de manera simulada procesos de entrenamiento quirúrgicos que permitan mejorar las capacidades de los cirujanos de forma segura, así como la medición de su progreso y evaluación de su experticia [16]. En el campo específico de las cirugías de tipo laparoscópico, se ha propiciado el desarrollo de sistemas de simulación médica que recrean algunos procedimientos quirúrgicos de gran complejidad [17].

\section{Sistemas GUIADOS Y ASISTIDOS}

La cirugía asistida por computador representa un concepto que engloba un conjunto de métodos, los cuales utilizan tecnología informática para la planificación pre quirúrgica y para orientar o realizar intervenciones en tiempo real, también conocida como intervención asistida por computador, cirugía guiada por imágenes o navegación quirúrgica. Los sistemas guiados permiten a los cirujanos en el preoperatorio, simular, educar y planear; y en el trans operatorio, guiar, asistir, ejecutar y decidir.

Una agrupación más amplia de los sistemas quirúrgicos guiados por computador presentados en [18] se muestra a continuación.

\section{A. Cirugía guiada}

La cirugía guiada se define como un sistema de navegación quirúrgica controlado por computador, con el que se puede guiar una ruta en un espacio de tres dimensiones [19]. En algunos casos se utilizan imágenes con sistemas de contraste, usando etiquetas o marcadores fluorescentes que permiten la navegación y orientación del sistema entre órganos y nervios con imágenes en tiempo real [20]. La cirugía guiada o asistida basada en computador abarca más que las acciones operatorias, y se relaciona generalmente con la planeación de la operación permitiendo mayor porcentaje de éxito dentro de los procedimientos [21].

Los métodos guiados pueden ser "estáticos" o "dinámicos". Los sistemas estáticos se basan en plantillas o guías para el flujo de trabajo de la cirugía, mientras que los sistemas dinámicos muestran el área de intervención y la posición en una interfaz de visualización o monitor; en estos casos se incluye navegación quirúrgica que permite ver la posición del instrumental en tiempo real [22] [23].

Los sistemas de navegación soportados en luz infrarroja han sido los más exitosos; su mecanismo de funcionamiento se sustenta en la localización de diodos emisores de infrarrojo a partir de tres cámaras ordenadas en forma lineal. Digi Pointeur es un ejemplo comercial de un sistema de navegación quirúrgica, cuya característica más relevante es la de usar marcadores electromagnéticos en vez de ópticos [24], y ha sido empleado en múltiples intervenciones mínimamente invasivas asociadas a la cabeza y cara, tales como implantes cocleares [25] implantología odontológica [26], extirpación de tumores intracraneales y monitoreo de los nervios faciales [27]; en tanto que en el área ortopédica, ORTHODOC, convierte imágenes de CT (tomografía computarizada) de cada paciente a una imagen virtual en 3D de la estructura de los huesos, que el cirujano puede manipular y usar como apoyo para la toma de decisiones en el plan quirúrgico [28].

Por su parte, Stealth Station es un sistema de navegación y planeación que se enfoca en el movimiento preciso de los instrumentos en relación con la anatomía del paciente [29]. NavSuite OR de Stryker es un sistema de asistencia por computador que utiliza una cámara montada en el techo, la cual hace seguimiento de los instrumentos e integra las imágenes de endoscopios, microscopios y fluoroscopios [30]. BrainLab VectorVision usa para la localización de dispositivos intra operatorios reflexión pasiva de flashes infrarrojos [31]. La estación Dash aprovecha la funcionalidad de herramientas tradicionales para crear mediciones que mejoren la precisión del instrumental y está diseñada para asistir en operaciones de rodilla; la aplicación software de esta estación de navegación permite que cualquier iPod funcione como monitor o despliegue [32].

\section{B. Cirugía asistida}

La cirugía robótica o cirugía asistida por computador (también llamada de tele presencia) se basa en un sistema interactivo computarizado. Según su participación en la intervención quirúrgica, este sistema puede dividirse en pasivos y activos. Se consideran pasivos aquellos que asisten al cirujano en alguna tarea de la operación; los activos son los que realizan la intervención directamente, comandados por la experticia de un cirujano humano. Algunos de los sistemas pasivos pueden caer dentro de la clasificación de sistemas guiados o de navegación quirúrgica [33], puesto que realizan cálculos y guían al cirujano durante la intervención, mientras que los activos se caracterizan porque el cirujano determina maniobras a distancia, las cuales son ejecutadas por un robot, en el paciente [34]. El profesional que opera en las cirugías asistidas con robots no tiene realimentación táctil y de fuerza, debido a que toca indirectamente la superficie de contacto, por lo cual surge la necesidad de incorporar la realimentación para mejorar la calidad de los procedimientos. Por esto se vienen desarrollando mecanismos que permiten 
diferenciar fácilmente entre órganos de distinta rigidez a partir de realimentación visual y de fuerza, obteniéndose mejores resultados en la mezcla de las dos realimentaciones que los obtenidos con una sola realimentación, ya sea solo de fuerza o solo visual [35].

\section{Cirugía simulada}

La cirugía simulada se basa en el uso de imágenes simuladas u objetos virtuales tanto de órganos como de instrumentos con determinadas propiedades físicas y visuales, tales objetos se obtienen después de procesar imágenes médicas reales como las tomografías computarizadas. El objetivo de este tipo de simulación es planear en el pre operatorio, procedimientos quirúrgicos reales o entrenar al cirujano para obtener mayor habilidad quirúrgica e identificar en determinadas cirugías, los posibles riesgos post operatorios en pacientes reales. Los beneficios del entrenamiento simulado han sido comprobados en algunos estudios para laparoscopia y procedimientos endovasculares [36].

Es importante destacar que el desarrollo exponencial de las técnicas mínimamente invasivas asistidas por robots, han impulsado los sistemas simulados para mejorar las destrezas quirúrgicas [37]. Existen diferentes opciones para la simulación de acciones quirúrgicas con fines educativos, como los simuladores basados en realidad virtual, realidad aumentada o virtualidad aumentada, sumados a los métodos tradicionales con animales vivos, cadáveres y modelos físicos. En cuanto a estos últimos, permiten practicar habilidades básicas de cirugía abierta y laparoscópica, lo que a su vez genera realismo sensorial, bajo costo y amplia disponibilidad, aunque su mayor desventaja está relacionada con un alto grado de subjetividad en los procesos de entrenamiento, ya que necesitan supervisión humana para medir su efectividad [38].

\section{1) Realidad virtual}

Uno de los campos de aplicación más interesantes de la realidad virtual está en el desarrollo de simuladores para MIS asistidos por robots. El objetivo principal de estos sistemas es el de entrenar a los cirujanos en las destrezas principales implicadas en procedimientos de telepresencia o de asistencia con robots.

Dependiendo en gran medida del robot y del procedimiento quirúrgico implicado, la literatura enuncia los siguientes procedimientos: uso de instrumental con 6 grados de libertad, entrenamiento de coordinación manoojo, entrenamiento de destrezas avanzadas como cortes y suturas, así como módulos específicos de entrenamiento, como colecistectomía, embarazos ectópicos, intervenciones de ovarios, nefrectomía transperitoneal e intervenciones MIS por un solo puerto [39].

Debido a que la realidad virtual (VR) tiene el potencial para estandarizar el entrenamiento con robots cirujanos, se han desarrollado cajas de entrenamiento para los robots quirúrgicos más populares como el Da Vinci. Tal es el caso del Da Vinci Skills Simulator (dVSS) [40] [41], un simulador de entrenamiento desarrollado conjuntamente con Mimic Technologies, el cual permite realizar ejercicios quirúrgicos que van desde lo básico a lo avanzado en casi cualquier especialidad.

En esta misma línea de interés se sitúa el dV-Trainer (DVT), uno de los entrenadores robóticos comerciales que se auto pondera como parte de los entrenadores con mayor número de ejercicios de entrenamiento, cubriendo desde el adiestramiento de cirujanos novatos a expertos; este entrenador se basa en la plataforma de simulación Msim [42] [43]. RoSS es otra caja de entrenamiento para cirugía mínimamente invasiva con asistencia robótica; usa realidad virtual para introducir al usuario en los fundamentos de la cirugía asistida con robots y opera bajo un currículum de múltiples niveles de diferente dificultad, guiando en los procesos de control y precisión con el uso de instrumentos por separado y en conjunto, control de la cámara, cortes y suturas, retracciones y disecciones [44].

Otro sistema es SEP Robot, entrenador de realidad virtual para cirugía con robots desarrollado por la empresa Sim Surgery. Consta de una consola conectada a dos instrumentos de siete grados de libertad, como los tendría un robot cirujano, con un dispositivo de seguimiento de movimiento que detecta la posición de los controles en el espacio y recrea esos movimientos en una imagen virtual mostrada en una pantalla; el sistema hace una grabación de la trayectoria de los instrumentos y determina el número de errores en la ejecución de cada tarea [45].

La gamificación de los juegos de realidad virtual también se ha sumado al entrenamiento quirúrgico, como es el caso de ProMIS, una estación de entrenamiento para cirugías laparoscópicas desarrollada por CAE Healthcare. En esta estación el usuario interactúa con modelos físicos y virtuales, se pueden realizar prácticas para múltiples incisiones de entrada o para cirugías de un solo puerto. Las simulaciones de ProMIS se basan en juegos de realidad virtual, seguimiento inteligente de marcadores y análisis de imágenes [46] [47].

LapSIM es otra estación de simulación para entrenamiento quirúrgico y contiene todas las herramientas hardware y software necesarias para el entrenamiento en varias intervenciones, permitiendo navegación de cámara, manejo de instrumentos, corte, levantamiento y agarre, sutura, precisión y velocidad, manejo de intestinos, disección fina, cierre y corte, e inserción de catéter usando o no una interfaz háptica [26].

\section{REALIDAD AUMENTADA}

La realidad aumentada es una técnica donde se amplían las imágenes médicas reales con información enriquecida que el cirujano no puede apreciar en el mundo real; dicha información es generada por computador y mostrada en un dispositivo de despliegue, fusionando las imágenes reales con las virtuales. Uno de los mayores retos de la realidad aumentada se encuentra en la sutileza de la realidad mixta, principalmente porque se desea que el usuario difícilmente diferencie el mundo real y el aumento virtual, teniendo la sensación de que se encuentra en la misma escena. La realidad aumentada (AR) se diferencia de la realidad virtual 
(VR) en que en la primera el usuario mantiene la sensación de presencia en el mundo real, mientras en la segunda se recrea un mundo virtual total.

La realidad aumentada tiene dos funciones: aumentar la percepción de la realidad (muestra la realidad, pero elige qué se puede ver y qué no), y la de crear un ambiente artificial (muestra lo que no es real permitiendo ver lo imaginario), con una percepción aumentada de información útil que ayuda a la toma de decisiones y acciones. Los sistemas de AR se caracterizan porque poseen elementos reales y virtuales en un entorno real con alto grado de interactividad, con la opción de registrar y posicionar la información virtual teniendo en cuenta la tridimensionalidad del mundo real.

Hay cuatro aspectos técnicos que se necesitan para integrar un sistema con AR, estos son: captación de la escena real, identificación, mezcla de realidad e información virtual, y visualización. En cuanto a los requerimientos hardware, se encuentran: computador o dispositivo móvil, monitor o dispositivo de despliegue, cámara, sistema de seguimiento o sensado, GPS, acelerómetro, infraestructura de conexión y marcadores, entre otros [48].

\section{A. Aplicaciones en la neurocirugía}

La simulación permite a los neurocirujanos en proceso de entrenamiento adquirir las competencias adecuadas en un campo crítico, donde los errores técnicos pueden tener resultados devastadores [49]. La simulación con realidad virtual tiene el potencial de jugar un papel importante dentro de los procesos de entrenamiento en la cirugía neurológica, por ejemplo, durante la remoción de tumores cerebrales [50]. A nivel comercial existe un número creciente de sistemas de simulación que posibilitan la manipulación virtual de una reconstrucción $3 \mathrm{D}$ a través de visualizaciones intracraneales, rotaciones, segmentaciones y otras operaciones, con el fin de generar un conocimiento detallado sobre una posible situación quirúrgica [17].

Algunos de los referentes bibliográficos muestran entrenamiento de neurocirugía con AR en procedimientos tan delicados como el puenteo extra-intra craneal, donde la información anatómica se integra en el campo quirúrgico y sirve de soporte en el desarrollo de un procedimiento mínimamente invasivo a partir de una ruta de trabajo preoperatorio [51] [52]. Otros sistemas como Neuro Touch incorporan escenarios simulados con métricas para determinar el nivel de manejo de diferentes habilidades quirúrgicas, tanto para cirujanos principiantes como expertos, en la remoción de tumores cerebrales [53].

En tanto que para entrenamientos con un enfoque más realista, se han desarrollado sistemas con seguimiento remoto de instrumentos y con despliegues estereoscópicos de alta resolución de píxeles, como Immersive Touch, donde el cirujano interactúa con un modelo anatómico virtual en 3D usando un instrumento háptico. Este sistema involucra entrenamientos tan complejos como la ventriculostomía, la cual consiste en el drenaje del fluido cerebro espinal a través de una apertura en uno de los ventrículos, entrenamiento en el cual Immersive Touch reproduce las sensaciones visuales táctiles y sonoras con alto realismo [54] [55].
En el caso de la navegación quirúrgica, en neurología se han implementado técnicas de realidad aumentada para visualizar de manera interactiva a través de un equipo computacional, la reconstrucción en tres dimensiones de una porción específica de un cerebro humano, y proyectando esta simulación sobre un modelo virtual [56]. En otras aplicaciones, la escena aumentada se ha creado sobreponiendo una trayectoria planeada en el preparatorio de una aguja con la ayuda de una malla de marcadores sobre el paciente, mientras el software AR proporciona una vista aumentada en tiempo real a través de un despliegue frontal ubicado en la cabeza del cirujano, todo esto dentro de un procedimiento de alto riesgo como es la vertebroplastia percutánea [57].

En el caso de la teleprescencia o cirugías remotas, se cuenta con el sistema VIPAR que permite comunicar una estación local y una remota, conectadas por internet interactuando en tiempo real [58]. Finalmente, existe un sistema para simulaciones de AR de alta precisión, donde se guían las agujas a partir de proyecciones con luz láser en la piel, conocido como Phantom, consistente en una proyección del recorrido de la aguja en las venas del paciente a partir de un apuntador láser [59].

\section{B. Aplicaciones en laparoscopia}

ProMIS AR es un ejemplo de simulador para entrenamiento en intervenciones laparoscópicas que hace uso de realidad aumentada y reúne las características de las estaciones de simulación más comunes. ProMIS AR emplea los instrumentos originales de laparoscopia y como software permite llevar medición del desempeño del cirujano [60]. Siguiendo la misma línea del anterior simulador, Maestro AR utiliza instrumentos virtuales y aumentación $3 \mathrm{D}$, esta vez presentando la aumentación sobre video. Es el componente de realidad aumentada para el entrenador dV-Trainer; en este sistema, el cirujano manipula instrumentos virtuales interactuando con regiones anatómicas de vistas reales y aumentaciones 3D en un video de cirugía real. Maestro AR identifica anatomía, anticipa retracciones y predice regiones de disección; el objetivo principal es refinar capacidades específicas de entrenamiento de cirugías en equipo [61].

Hands-On Surgical Training (HoST) es el componente de realidad aumentada para el entrenador de cirugía asistida por robot denominado RoSS; este sistema guía al cirujano a través de una secuencia de aprendizaje, en la cual solo se puede avanzar después de haber realizado satisfactoriamente cada paso de la cirugía. Dicho entrenador trabaja grabando y reproduciendo los movimientos correctos de la mano del cirujano que manipula unos instrumentos virtuales sobre videos de casos quirúrgicos reales [62].

Por su parte, ARH consiste básicamente en un driver Linux y un protocolo de calibración para adquirir los datos convencionales de las herramientas de laparoscopia; para el funcionamiento en tiempo real usa Polhemus Isotrack [63], el cual es un software de seguimiento o tracking que permite determinar posición y orientación de objetos específicos, usado en este caso para el seguimiento de los movimientos de las herramientas laparoscópicas [64]. 
Las proyecciones láser de realidad aumentada no solo se limitan a casos externos y sobre el cuerpo del paciente. Pico Lantern es un proyector láser muy pequeño que se introduce en la zona abdominal y permanece allí durante la operación laparoscópica, proyectando sobre los órganos internos una grilla que permite que el médico tenga una percepción de volumen y proporción en la imagen $2 \mathrm{D}$ proporcionada por la cámara laparoscópica [65].

En el campo de la laparoscopia la complejidad es mayor respecto a las intervenciones neurológicas, ya que en el abdomen la deformación debido a la respiración es mayor. Los pasos claves en este tipo de intervenciones son el seguimiento del instrumental quirúrgico, la adquisición de la imagen 3D, el registro de dicha imagen en el campo quirúrgico y la intermediación entre el intervalo de tiempo requerido para sobreponer la imagen $3 \mathrm{D}$ en la vista endoscópica [66].

Uno de los trabajos más sobresalientes en este campo es el uso de la realidad aumentada en una pancreatectomía a partir de imágenes de tomografía computarizada. En este caso se hace la reconstrucción 3D del órgano con segmentación, y el registro inicial o tracking se realiza con un sensor de localización óptico, obteniéndose errores mínimos de precisión [67].

En operaciones de irradiación localizada con radiofrecuencia para el tratamiento del cáncer hepático, se utiliza un procedimiento que consiste en introducir una aguja hasta el tumor y aplicar una inyección de radiofrecuencia para hacer morir el tejido canceroso por hipertermia. La acción de ubicar la aguja en el sitio cercano al tumor es una tarea de alta dificultad, ya que para la guía se usa ultrasonido, tomografía computarizada o imágenes de resonancia magnética. En este caso, los sistemas de realidad aumentada ayudan en la ubicación de la aguja y en las tareas de planeación preoperatoria, gracias a la visualización de modelos 3D de los órganos del paciente reconstruidos a partir de imágenes médicas [68].

\section{Aplicaciones en ortopedia y odontología}

Entre los entrenadores basados en realidad aumentada se han desarrollado sistemas que permiten a los estudiantes de medicina efectuar una primera aproximación al procedimiento de acceso venoso central en recién nacidos. Este es el caso de un sistema desarrollado en la Universidad Militar Nueva Granada en Colombia, el cual posee herramientas para el seguimiento de posición y orientación de un marcador 3D, lo que permite al usuario interactuar con modelos de herramientas quirúrgicas tales como la jeringa, alambre guía, dispositivo de dilatación y el catéter, cada uno de ellos superpuestos como contenido virtual sobre el marcador. Este sistema también dispone de entradas de teclado con el fin de desplazarse en la escena y cambiar entre las vistas de la piel, el esqueleto o el sistema circulatorio del paciente. El prototipo está programado en Unity3D con el uso de la librería para RA Vuforia y un Oculus VR con una cámara web adjunta [69].

También se registra el implemento de un sistema para intervenciones endodónticas, consistente en un software que usa $\mathrm{C}++$, Qt y la librería de procesamiento de imágenes de OpenCV, en la cual el diente intervenido es detectado en imágenes de video de una cámara intraoral, y a partir de un algoritmo de identificación se lleva a cabo el reconocimiento del canal de raíces del diente. Esta información se sobrepone en la imagen real, donde la localización, el tamaño, la orientación y las distancias son guardadas para posteriores estudios morfológicos [70].

En el caso de la cirugía ortopédica y de trauma, la tecnología AR se constituye en una ayuda en el cambio de tareas y en el entendimiento de la relación entre la anatomía, los implantes y las herramientas.

Al respecto, se han realizado trabajos que permiten una visualización de la escena operatoria que mezclan las diferentes fuentes de información, entre ellas los datos proporcionados por un sensor Kinect. En este desarrollo se introduce un paradigma basado en aprendizaje de máquina, en el cual se identifican aspectos relevantes de la anatomía con el Kinect y los datos de rayos X por un lado, y por otro lado, se crea un mapa que mezcla las imágenes en video junto a las imágenes de rayos $\mathrm{X}$ en una sola vista, obteniendo excelentes resultados en el reconocimiento de la escena quirúrgica y la ampliación del campo de percepción [71].

Finalmente está ARDental, un sistema de realidad aumentada construido por varias universidades para ayudar al entrenamiento de intervenciones en el campo odontológico; combina elementos reales y modelos $3 \mathrm{D}$, lo cual resulta revolucionario en el sentido de que este tipo de entrenamiento se realizaba anteriormente a partir de imágenes 2D [72].

\section{Interfaces}

Dentro de las interfaces para realidad aumentada más relevantes y recientes se tiene See-through, un tipo de interfaz que funciona básicamente a través de un arreglo de espejos ubicados al frente de los ojos del usuario, quien podrá ver el mundo real y por reflexión las imágenes virtuales producidas por los monitores. Algunos ejemplos de esta tecnología se pueden encontrar en simuladores quirúrgicos y en la superposición de imágenes de ultrasonido en un paciente real. También se han realizado aplicaciones de procedimientos médicos integrando Google glass con el Virtual Interactive Presence and Augmented Reality (VIPAR), para cirugías remotas [73].

Gracias a la invención del primer HMD (Head-mounted Display) se introdujo el concepto de realidad aumenta en 1960 por Ivan Sutherland [74] y desde entonces se ha realizado variaciones para permitir una mayor inmersión del usuario. La inmersión e integración del mundo real y simulado del HMD proporciona una percepción bastante particular a los usuarios, quienes tienen la posibilidad de ver, sentir y oler, y con muy poco margen, distinguir entre lo real y lo generado por computador [75].

Otra interface son los despliegues por proyección, que surgen como una alternativa para aprovechar proyecciones con el fin de mostrar la información directamente sobre los objetos del mundo físico, y a partir de eso conseguir el objetivo de aumentación. El despliegue se hace con un 
proyector monoscópico y no necesita de otro dispositivo especial para ser visto [76].

De igual modo, las Interfaces hápticas surgen como respuesta a los grandes retos de recrear las sensaciones tácticas del instrumental. Uno de los más recientes avances en estos dispositivos es REVEL, que permite obtener una sensación táctil de textura sin necesidad de guantes; se basa en el efecto de electro vibración, para lo cual se usa un pico de señal eléctrica creando una oscilación eléctrica alrededor de los dedos del usuario, recreando la sensación de textura [77]. También se realizan trabajos para estimulación háptica virtual a partir de la háptica real, teniendo en cuenta la rigidez de los objetos, desarrollo que ha sido aplicado a entrenadores para la palpación de tumores [78].

\section{DISCUSIÓN}

La literatura menciona que una de las principales ventajas de la utilización de un sistema de entrenamiento virtual en cirugías de alto riesgo o alta complejidad, es permitir comparar el desempeño de cirujanos expertos respecto a los estudiantes en formación en cuanto a sus habilidades psicomotoras [50]. En estudios comparativos entre cajas de simulación con realidad aumentada como la ProMIS, y cajas de realidad virtual como la LapSim, los resultados son ligeramente mejores en la estación de simulación con realidad aumentada al haberse realizado la prueba con grupos de médicos novatos y expertos. Esta situación se presente debido principalmente al realismo en la realimentación háptica, la cual resulta ser un reto en los aparatos de realidad virtual, mientras que en los sistemas de realidad aumentada el médico opera instrumentos reales, tal como los tendría en una operación laparoscópica real [79].

Los estudios también arrojan que sistemas de entrenamiento con realidad virtual presentan mejores resultados frente a los simuladores físicos, pero la VR tiene una limitación en la adquisición de percepción visual [80], por lo cual algunos artículos sugieren enfocar las investigaciones en la implementación de procedimientos complejos, generación de efecto sinérgico entre la simulación VR y el entrenamiento mental (manejo del estrés por ejemplo), estandarización del entrenamiento, $\mathrm{y}$ entrenamiento personalizado [81].

Los avances de AR no se limitan al campo visual sino también al táctil, recreando sensaciones táctiles virtuales, lo cual abre camino a la realidad aumentada aplicada a la háptica $\mathrm{o} A \mathrm{AR}$ háptica, vislumbrando nuevos panoramas de investigación dirigidos a la mezcla de sensaciones del mundo real y virtual, y ampliando sensaciones como fuerza, rigidez, flexibilidad, rugosidad, temperatura y demás [78].

En cuanto a la ampliación de la percepción visual en entrenadores y ayudantes con AR, actualmente se realizan trabajos en la mejora de la ampliación de la percepción del campo visual en AR alternando con VR, lo que facilita la tarea quirúrgica [82]. Se trabaja además sobre el realismo de la simulación híbrida, con el fin de simular el corte en tiempo real, así como la deformación y realimentación para el entrenamiento quirúrgico [83].

Algunas revisiones sistemáticas han concluido que aspectos técnicos como el seguimiento y registro son los temas más importantes para aplicaciones de AR. A su vez, diversos estudios han arrojado luz sobre el proceso de seguimiento de la realidad aumentada, pero no se han reportado con claridad aún los desafíos y limitaciones de estos procesos [84] [85]. Por tal razón, una de las áreas de mayor desarrollo en el futuro será el seguimiento y la localización espacial de la posición de los instrumentos quirúrgicos en tiempo real. También, muchos trabajos proponen el uso de sensores magnéticos y sensores ópticos, incluyendo también ultrasonido y tomografía computarizada preoperatoria, o imágenes de resonancia magnética para dicho seguimiento [61], mientras que otros estudios mencionan problemas relevantes como la exactitud, la portabilidad y el costo [60].

Otro de los problemas por resolver en este campo se refiere al seguimiento de marcadores, ya que una de las grandes limitaciones de los sistemas de AR en operaciones intra abdominales son las deformaciones debido a la respiración del paciente, para lo cual es necesario un sistema de seguimiento dinámico del movimiento de los órganos abdominales [61].

Por último, la combinación de la realidad aumentada en tiempo real y los dispositivos portátiles (wearable computing devices) como Google glass, es una tendencia en el campo de la cirugía [61]. Este será uno de los vectores de desarrollo futuro, ya que el despliegue en los sistemas de realidad aumentada sigue siendo un factor limitante [86]. Actualmente se trabaja mucho en despliegues que proporcionan suficiente brillo, resolución, campo visual y contraste, para apreciar el mundo real e imaginario. Las tecnologías propuestas aún presentan muchas limitantes y no son lo suficientemente pequeñas, livianas ni de bajo costo [75].

\section{CONCLUSIONES}

A través de este artículo se presentó una revisión sobre los principales avances en realidad aumentada, de lo cual se concluye que existe un creciente uso de esta tecnología dentro del área quirúrgica, no solo en las estaciones de entrenamiento sino también dentro de las cirugías guiadas, apoyando el trabajo de los cirujanos. Esto se debe principalmente a la creación de imágenes $3 \mathrm{D}$ a partir de imágenes médicas en 2D y datos médicos en tiempo real, que llevan a recrear un ambiente con mayor información visual para el cirujano, permitiéndole tomar mejores decisiones dentro de las intervenciones quirúrgicas.

De esta revisión también se concluye que son varios los estudios que arrojan como resultado el beneficio de la simulación en el entrenamiento quirúrgico y en la cirugía guiada, y que el impacto de los entrenadores de realidad aumentada (AR) en aspectos tales como la realimentación háptica, resulta ser mayor sobre los de realidad virtual (VR).

En el caso de los simuladores, las aplicaciones para estaciones de entrenamiento se han enfocado en recrear instrumentos virtuales sobre videos de operaciones reales, al tiempo que el cirujano usa instrumentos similares a los que $u$ Los estudios muestran que las investigaciones futuras en simulación y entrenamiento con AR se dirigen, por un lado, hacia el realismo visual debido al papel limitante de 
recrear un mundo cercano a la realidad y, por otro lado, hacia la realimentación háptica, así como hacia el seguimiento dinámico de marcadores y órganos para una aumentación con alto grado de afinidad con la realidad.

En cuanto a las aplicaciones de aumentación en AR en cirugía mínimamente invasiva, los trabajos consultados muestran que la realidad aumentada no solo se ha limitado al área visual, pues en la actualidad se abarcan algunos desarrollos de aumentación en lo táctil y lo auditivo, para recrear las sensaciones de una cirugía abierta. Una de las aplicaciones de aumentación más interesantes es la de proyectar sobre el paciente imágenes, o sobre los órganos, ya sea venas o cuadrículas, que servirán de guía al cirujano con agujas, catéteres y cortes.

Dentro de las áreas de investigación en AR, el seguimiento y la localización espacial de la posición de los instrumentos quirúrgicos resulta ser tema de tendencia investigativa, así como el seguimiento o tracking en operaciones intraabdominales, debido al efecto de la respiración que requiere de un sistema de seguimiento dinámico del movimiento de los órganos en anatomías específicas.

\section{REFERENCIAS}

[1] Nicolau, S., Soler, L., Mutter, D. and Marescaux, J., "Augmented reality in laparoscopic surgical oncology." Surgical oncology, vol. 20, no. 3, pp. 189-201, 2011.

[2] Yu, H. Y., Hevelone, N. D., Lipsitz, S. R., Kowalczyk, K. J. and Hu, J. C., "Use, costs and comparative effectiveness of robotic assisted, laparoscopic and open urological surgery", The Journal of urology, 187(4), pp. 1392-1399, 2012.

[3] Miccoli, P., Bellantone, R., Mourad, M., Walz, M., Raffaelli, M. and Berti, P. "Minimally invasive video-assisted thyroidectomy: multiinstitutional experience," World journal of surgery, vol. 26, no. 8, pp. 972-975, 2002.

[4] Van der Pas, M., Haglind, E., Cuesta, M. A., F urst, A., Lacy, A.M., Hop, W. C., Bonjer, H. J., Cancer Laparoscopic or Open Resection II(COLOR II) Study Group, et al., "Laparoscopic versus open surgery for rectal cancer (color ii): short-term outcomes of a randomised, phase 3 trial", The lancet oncology, vol. 14, no. 3, pp. 210-218, 2013.

[5] Pugliese, R. and Bailey, M., "Laparoscopic surgery: the need of training centres to spread knowledge," Journal of Medicine and The Person, vol. 6, no. 4, pp. 160-163, 2008.

[6] Marescaux, J. and Diana, M., "Next step in minimally invasive surgery:hybrid image-guided surgery", Journal of pediatric surgery, vol. 50, no. 1, pp. 30-36, 2015.

[7] Jamali, S. S., Shiratuddin, M. F., Wong, K. W. and Oskam, C. L., "Utilising mobile-augmented reality for learning human anatomy," Procedia-Social and Behavioral Sciences, vol. 197, pp. 659-668, 2015.

[8] Han, P. and Zhao, G., "Cad-based 3d objects recognition in monocular images for mobile augmented reality", Computers \& Graphics, vol 50, pp. 36-46, 2015.

[9] Wen, R., Tay, W. -L., Nguyen, B. P., Chang, C. -B. and Chui, C. $-\mathrm{K}$., "Hand gesture guided robot-assisted surgery based on a direct augmented reality interface", Computer methods and programs in biomedicine, vol. 116, no. 2, pp. 68-80, 2014.

[10] Guzmán, D. E. and Albán O. A., "Software para la práctica de la robótica quirúrgica", Ingeniería y Universidad, vol. 19, no. 1, pp. 7-26, 2015.

[11] Moreno, M. R., Moraes, T. F., Amorim, P. H., da Silva, J. V. L. and Rodríguez, C. A., "Virtual open source environment for training and simulation of laparoscopic surgery", in XII Workshop de Informática Médica (WIM'2012)-XXXII Congresso da Sociedade Brasileira de Computaçao, pp. 1-4, 2012.

[12] Lacy, de A. M., Rattner, D. W., Adelsdorfer, C., Tasende, M.M.,
Fernández, M., Delgado, S., Sylla, P. and Martínez-Palli, G., "Transanal natural orifice transluminal endoscopic surgery (notes) rectal resection: "down-to-up" total mesorectal excision (tme) shortterm out comes in the first 20 cases", Surgical endoscopy, vol. 27, no. 9, pp. 3165-3172, 2013.

[13] Autorino, R., Cadeddu, J. A., Desai, M. M., Gettman, M., Gill, I. S., Kavoussi, L. R., Lima, E., Montorsi, F., Richstone, L., Stolzenburg, L. U., et al., "Laparoendoscopic single-site and natural orifice transluminal endoscopic surgery in urology: a critical analysis of the literature", European urology, vol. 59, no. 1, pp. 26-45, 2011.

[14] Rané, A. and Rao, P., "Single-port-access nephrectomy and other laparoscopic urologic procedures using a novel laparoscopic port (r-port)", Urology, vol. 72, no. 2, pp. 260-263, 2008.

[15] Autorino, R., Kaouk, J. H., Yakoubi, R., Rha, K. H., Stein, R. J., White, W. M., Stolzenburg, J. -U., Cindolo, L., Liatsikos, RaisBahrami, S., et al., "Urological laparoendoscopic single site surgery: multi-institutional analysis of risk factors for conversion and postoperative complications", The Journal of urology, vol. 187, no. 6, pp. 1989-1994, 2012.

[16] Duchene, D. A., Moinzadeh, A., Gill, I. S., Clayman, R. V. and Winfield, H. N., "Survey of residency training in laparoscopic and robotic surgery", The Journal of urology, vol. 176, no. 5, pp. 21582167, 2006.

[17] Benítez, P. A., "Desarrollo de un sistema de realidad aumentada para entrenamiento en planificación neuroquirúrgica", tesis para optar al grado de magíster en ciencias de la ingeniería electrónica con mención en computadores, Universidad técnica Federico Santa María Departamento de Electrónica Valparaíso, Chile, 2014.

[18] Mosso, J. L., "Propuesta de una clasificación quirúrgica", Cirugía y Cirujanos, vol 71, pp. 492-495, 2003.

[19] Melkent, T., Foley, K. T., Estes, B. T. and Chaudoin, J., "Image guided spinal surgery guide system and method for use thereof", U.S. Patent 8,105,339, issued January 31, 2012.

[20] Nguyen, Q. T., Tsien. R. Y., "Fluorescence-guided surgery with live molecular navigation [mdash] a new cutting edge", Nature Reviews Cancer 13, no. 9, pp. 653-662, 2013.

[21] Huang, D., He, D. -m, Yang, C., Chen, M. -j., Zhou, Q. and Dong, M. -j., "Computer-assisted local resection for exostosis osteochondroma of the mandibular condyle", Journal of Craniofacial Surgery, 24, no. 4, pp. e446-e449, 2013.

[22] Vercruyssen, M., Laleman, I., Jacobs, R. and Quirynen, M., "Computer-supported implant planning and guided surgery: a narrative review", Clinical oral implants research, vol. 26, no. S11, pp. 69-76, 2015.

[23] Torresanto, di V. M., Milinkovic, I., Torsello, F. and Cordaro, L., "Cirugía implantológica sin colgajo, asistida por ordenador, en pacientes edéntulos de edad avanzada: seguimiento de 2 años", Quintessence: Publicación internacional de odontología, vol. 2, no. 5, pp. 318-329, 2014.

[24] Lasserre, F. and Auber, N., "Navigation chirurgicale associée à l'imagerie per-opératoire", IRBM news, vol. 30, no. 4, pp. 15-29, 2009.

[25] Nguyen, Y., Miroir, M., Vellin, J. -F, Mazalaigue, S., Bensimon, J. -L, Bernardeschi, D., Ferrary, E., Sterkers, O. and Grayeli, A. B., "Minimally invasive computer-assisted approach for cochlear implantation a human temporal bone study", Surgical innovation, vol. 18, no. 3, pp. 259-267, 2011.

[26] Bernardeschi, D., Nguyen, Y., Villepelet, A., Ferrary, E., Mazalaigue, S., Kalamarides, M. and Sterkers, O., "Use of bone anchoring device in electromagnetic computer-assisted navigation in lateral skull base surgery", Acta oto-laryngologica, vol. 133, no. 10, pp. 1047-1052, 2013.

[27] Collin, M., Bernardeschi, D., Cazals-Hatem, D. and Sterkers, O., "Menin-gioma of geniculate ganglion: case report and review of the literature", Acta oto-laryngologica, vol. 133, no. 3, pp. 228-232, 2013.

[28] Prnasky J. "ROBODOC-surgical robot success story", Industrial Robot: An International Journal, vol 24, no 3, pp 231-233, 1997.

[29] Molloy, S., Aftab, S., Patel, A., Butler, J., Balaji, V., Wilson, L. and Lee, R., "The british experience of pedicle screw insertion using the o-arm imaging system and stealth station navigation system", Bone \& Joint Journal Orthopaedic Proceedings Supplement, vol. 96, no. 
SUPP 15, pp. 30-30, 2014.

[30] Xiaojun C., "Development of a surgical navigation system based on augmented reality", Journal of Biomedical Informatics, no. 55, pp. 124-131, 2015.

[31] Gerbino, G., Zavattero, E. Berrone, M. and Berrone, S., "Management of needle breakage using intraoperative navigation following inferior alveolar nerve block", Journal of Oral and Maxillofacial Surgery, vol. 71, no. 11, pp. 1819-1824, 2013.

[32] Mezger, U., Jendrewski, C. and Bartels, M., "Navigation in surgery," Langenbeck's Archives of Surgery, vol. 398, no. 4, pp. 501-514, 2013.

[33] Kantelhardt, S. R., Greke, C., Keric, N., Vollmer, F., Thiemann, I. and Giese, A., "Image guidance for transcranial doppler ultrasonography", Neurosurgery, vol. 68, pp. ons257-ons266, 2011.

[34] García, O., Olvera, H. and Beltrán, J., "Telemedicina y cirugía robótica en ginecología", Ginecol Obstet Mex, vol. 76, no. 3, pp. 161-166, 2008

[35] Tholey, G., Desai, J. P. and Castellanos, A. E., "Force feedback plays a significant role in minimally invasive surgery: results and analysis", Annals of surgery, vol. 241, no. 1, p. 102, 2005.

[36] Robinson, W. P., Schanzer, A., Cutler, B. S., Baril, D. T., Larkin, A. C., Eslami, M. H., Arous, E. J. and Messina, L. M., "A randomized comparison of a 3-week and 6-week vascular surgery simulation course on junior surgical resident performance of an end-to-side anastomosis", Journal of vascular surgery, vol. 56, no. 6, pp. 17711781, 2012.

[37] Perrenot, C., Perez, M., Tran, N., Jehl, J. -P., Felblinger, J., Bresler, L. and Hubert, J., "The virtual reality simulator dv-trainer is a valid assessment tool for robotic surgical skills", Surgical endoscopy, vol. 26, no. 9, pp. 2587-2593, 2012.

[38] Jakimowicz, J. J. and Jakimowicz, C. M., "Simulación en cirugía, ¿dónde estamos y a dónde llegaremos?”, Cir Cir, vol. 79, pp. 44-49, 2011.

[39] Sonja, N. Buzink, Richard H.M. Goossens, Huib De Ridder \& Jack J. Jakimowicz (2010) Training of basic laparoscopy skills on SimSurgery SEP, Minimally Invasive Therapy \& Allied Technologies, vol.19, no. 1, pp. 35-41, 2010.

[40] Bric, D., Lumbard, D. C., Frelich, M. J. and Gould, J. C., "Current state of virtual reality simulation in robotic surgery training: a review", Surgical endoscopy, vol. 30, no. 6, pp. 1-10, 2015.

[41] Guthart, G. and Salisbury, J., The Intuitive/sup TM/telesurgery system: overview and application, Robotics and Automation, 2000, ICRA'00 IEEE International Conference, vol. 1, pp. 618-621, 2000.

[42] Perrenot, C., Perez, M., Tran, N., Jehl, J., Felblinger, J., Bresler, L., and Hubert, J., The virtual reality simulator $\mathrm{dv}$-trainerR $\odot$ is a valid assessment tool for robotic surgical skills. Surgical endoscopy, vol. 26, no. 9, pp. 2587-2593, 2012.

[43] Hung, A. J., Jayaratna, I. S., Teruya, K., Desai, M.M., Gill, I. S. and Goh, A. C., "Comparative assessment of three standardized robotic surgery training methods", BJU international, vol. 112, no. 6, pp. 864-871, 2013.

[44] Simulated Surgical Systems: Products", Simulated Surgical Systems, http://www.simulatedsurgicals.com/ross2.html, Accessed: 28- Sep- 2018.

[45] Gavazzi, A., Bahsoun, Van Haute, W. V., Ahmed, K., Elhage, O., Jaye, P., Khan, M. S. and Dasgupta, P., "Face, content and construct validity of a virtual reality simulator for robotic surgery (SEP Robot)". Annals of the Royal College of Surgeons of England, vol. 93, no. 2, pp. 152-156, 2011.

[46] Abboudi, H., Khan, M., Aboumarzouk, O., K. Guru, A., Challacombe, B., Dasgupta, P., and Ahmed, K., "Current status of validation for roboticsurgery simulators-a systematic review,"BJU international, vol. 111, no. 2, pp. 194-205, 2013.

[47] Botden, S., Buzink, S., Schijven, M., and Jakimowicz, J., "Promisaugmented reality training of laparoscopic procedures face validity,"Simulation in healthcare, vol. 3, no. 2, pp. 97-102, 2008.

[48] Xiao, D., Jakimowicz, J. J., Albayrak, A., Buzink, S. N., Botden, S. M. and Goossens, R. H., "Face, content, and construct validity of a novel portable ergonomic simulator for basic laparoscopic skills", Journal of surgical education, vol. 71, no. 1, pp. 65-72, 2014.

[49] Kipper, G. and Rampolla, J. Augmented Reality: an emerging technologies guide to AR. Elsevier, 2012.
[50] Kirkman, M., Ahmed, M., Albert, A., Wilson, M., Nandi, D., and Sevdalis, N., "The use of simulation in neurosurgical education and training: A systematic review,"Journal of neurosurgery, vol. 121, no. 2, pp. 228-246,2014.

[51] AlZhrani, G., AlOtaibi, F., Azarnoush, H., Winkler-Schwartz, A., Sabbagh, A., Lajoie, S. and Del Maestro, R., "Ms-05 utilizing a virtual reality simulator,neurotouch, to determine proficiency performance benchmarks for resection of simulated brain tumors", Neuro-Oncology, vol. 16, no. suppl 5, pp. 127-128, 2014.

[52] Azarnoush, H., Alzhrani, G., Winkler-Schwartz, A., Alotaibi, F., Gelinas-Phaneuf, N., Pazos, V., Choudhury, N., Fares, J., DiRaddo, R. and Del Maestro, R. F., "Neurosurgical virtual reality simulation metrics to assess psychomotor skills during brain tumor resection", International journal of computer assisted radiology and surgery, vol. 10, no. 5, pp. 603-618, 2014.

[53] Cabrilo, I., Schaller, K. and Bijlenga, P., "Augmented realityassisted bypass surgery: Embracing minimal invasiveness", World neurosurgery, vol. 83, no. 4, pp. 596-602, 2015.

[54] Alotaibi, F. E., AlZhrani, G. A., Mullah, M.A., Sabbagh, A. J., Azarnoush, H., Winkler-Schwartz, A. and Del Maestro, R. F., "Assessing bimanual performance in brain tumor resection with neurotouch, a virtual reality simulator", Operative Neurosurgery, vol. 11, no. 1, pp. 89-98, 2015.

[55] Alaraj, A, Luciano, C. J., Bailey, D. P., Elsenousi, A., Roitberg, B. Z., Bernardo, A., Banerjee, P. P. and Charbel, F. T., "Virtual reality cerebral aneurysm clipping simulation with real-time haptic feedback", Operative Neurosurgery, vol. 11, no. 1, pp. 52-58, 2015

[56] Yudkowsky, R., Luciano, C., Banerjee, P., Schwartz, A., Alaraj, A., Lemole Jr, G, F., Charbel, F., Smith, K., Rizzi, S., Byrne, R., et al., "Practice on an augmented reality/haptic simulator and library of virtual brains improves residents' ability to perform a ventriculostomy", Simulation in Healthcare, vol. 8, no. 1, pp. 2531, 2013.

[57] Glossop, N., Wedlake, C., Moore, J., Peters, T. and Wang, Z., Laser projection augmented reality system for computer assisted surgery. In Medical Image Computing and Computer-Assisted InterventionMICCAI 2003 (pp. 239-246). Springer Berlin Heidelberg, 2003.

[58] Abe, Y., Sato, S., Kato, K., Hyakumachi, T., Yanagibashi, Y., Ito, M. and Abumi, K., "A novel 3d guidance system using augmented reality for percutaneous vertebroplasty: technical note", Journal of Neurosurgery: Spine, vol. 19, no. 4, pp. 492-501, 2013.

[59] Shenai, M. B., Dillavou, M., Shum, C., Ross, D., Tubbs, R. S., Shih, A. and Guthrie, B. L., "Virtual interactive presence and augmented reality (vipar) for remote surgical assistance", Neurosurgery, vol. 68, pp. ons200-ons207, 2011.

[60] Ungi, T., Yeo, C. T., Paweena, U., McGraw, R. C., Fichtinger, G., et al., "Augmented reality needle guidance improves facet joint injection training", in SPIE Medical Imaging, pp. 79642E-79642E, International Society for Optics and Photonics, 2011.

[61] Harris, A., Bello, F. and Kneebone, R., "Simulation and training in minimal access surgery", in Training in Minimal Access Surgery, pp. 35-47, Springer, 2015.

[62] Maestro AR - Mimic Simulation: 2018. http://mimicsimulation. com/maestro-ar/. Accessed: 2018- 09- 28

[63] Simulated Surgical Systems introduces HoST: 2018. http://www. simulatedsurgicals.com/host/. Accessed: 2018- 09- 28.

[64] Polhemus Markets - Healthcare: 2018. https://polhemus.com/ markets/healthcare/. Accessed: 2018- 09- 28.

[65] Pagador, J. B., Sánchez, L., Sánchez, J., Bustos, P., Moreno, J. and Sánchez-Margallo, F. M., "Augmented reality haptic (arh): an approach of electromagnetic tracking in minimally invasive surgery", International journal of computer assisted radiology and surgery, vol. 6, no. 2, pp. 257-263, 2011.

[66] Edgcumbe, P., Pratt, P., Yang, G -Z, Nguan, C. and Rohling, R., "Pico lantern: Surface reconstruction and augmented reality in laparoscopic surgery using a pick-up laser projector", Medical Image Analysis, vol. 25, no. 1, pp. 95-102, 2015.

[67] Ukimura, O. and Gill, I. S., "Imaging-assisted endoscopic surgery: Cleveland clinic experience", Journal of Endourology, vol. 22, no. 4, pp. 803-810, 2008.

[68] Bruellmann, D., Tjaden, H., Schwanecke, U. and Barth, P., "An optimized video system for augmented reality in endodontics: a 
feasibility study", Clinical oral investigations, vol. 17, no. 2, pp. 441-448, 2013.

[69] Okamoto, T., Onda, S., Yasuda, J., Yanaga, K., Suzuki, N. and Hattori, A., "Navigation surgery using an augmented reality for pancreatectomy", Digestive surgery, vol. 32, no. 2, pp. 117-123, 2015.

[70] Gutiérrez, E. M., "Sistema de realidad aumentada para la interacción con el instrumental en el procedimiento de acceso venoso central", Informe final de auxiliar de Investigación para optar al Título de Ingeniero en Mecatrónica, Universidad Militar Nueva Granada, Colombia, 2015.

[71] De Paolis, L. T., Ricciardi, F., Dragoni, A. F. and Aloisio, G., "An augmented reality application for the radio frequency ablation of the liver tumors", in Computational Science and Its ApplicationsICCSA, pp. 572-581, Springer, 2011.

[72] Pauly, O., Diotte, B., Fallavollita, P., Weidert, S., Euler, E. and Navab, N., "Machine learning-based augmented reality for improved surgical scene understanding", Computerized Medical Imaging and Graphics, vol. 41, pp. 55-60, 2015.

[73] Folguera, F., Juan, M. C., Herrero, A. and Alexandrescu, L., "Introducción a una nueva dimensión en la morfología dentaria: Ardental (realidad aumentada dental)", Gaceta dental: Industria y profesiones, no. 252, pp. 200-209, 2013.

[74] Lindeque, B. G., Ponce, B. A., Menendez, M. E., Oladeji, L. O., Fryberger, C. T. and Dantuluri, P. K., "Emerging technology in surgical education: combing in greal-time augmented reality and wearable computing devices", Orthopedics (Online), vol. 37, no. 11, p. 751, 2014.

[75] Sutherland, I. E., "A head-mounted three dimensional display", in Proceedings of the December 9-11, 1968, fall joint computer conference, part I, pp. 757-764, ACM, 1968.

[76] Taqvi, Z., "Reality and perception: Utilization of many facets of augmented reality", in Artificial Reality and Telexistence (ICAT), 23rd International Conference on, pp. 11-12, IEEE, 2013.

[77] Pandya, A., "Augmented reality," International Journal of Research in Advance Engineering, vol. 1, no. 1, pp. 10-19, 2015.

[78] Bau, O. and Poupyrev, I., "Revel: tactile feedback technology for augmented reality", ACM Transactions on Graphics (TOG), vol. 31, no. 4, p. 89, 2012.

[79] Jeon, S. and Harders, M., "Extending haptic augmented reality: Modulating stiffness during two-point squeezing", in Haptics Symposium (HAPTICS), 2012 IEEE, pp. 141-146, IEEE, 2012.

[80] Botden, S. M., Buzink, S. N., Schijven, M. P. and Jakimowicz, J. J., "Augmented versus virtual reality laparoscopic simulation: what is the difference?", World Journal of Surgery, vol. 31, no. 4, pp. 764-772, 2007.

[81] Nomura, T., Mamada, Y., Nakamura, Y., Matsutani, T., Hagiwara, N., Fujita, I., Mizuguchi, Y., Fujikura, T., Miyashita, M. and Uchida, E., "Laparoscopic skill improvement after virtual reality simulator training in medical students as assessed by augmented reality simulator", Asian journal of endoscopic surgery, vol. 8, no. 4, pp. 408-412, 2015.

[82] Yiannakopoulou, E., Nikiteas, N., Perrea, D. and Tsigris, C., "Virtual reality simulators and training in laparoscopic surgery", International Journal of Surgery, vol. 13, pp. 60-64, 2015.

[83] Choi, H., Cho, B., Masamune, K., Hashizume, M. and Hong, J., “An effective visualization technique for depth perception in augmented reality-based surgical navigation", The International Journal of Medical Robotics and Computer Assisted Surgery, vol. 12, no. 1, pp. 62-72, 2016.

[84] Cotin, S., Delingette, H. and Ayache, N., "A hybrid elastic model for real-time cutting, deformations, and force feedback for surgery training and simulation", The Visual Computer, vol. 16, no. 8, pp. 437-452, 2000.

[85] Rabbi, I., Ullah, S. and Khan, S. U., "Augmented reality tracking techniques-a systematic literature", IOSR Journal of Computer Engineering (IOSRJCE), vol. 2, no. 2, pp. 23-29, 2012.

[86] Jeon, S., Choi, S. and Harders, M., "10 haptic augmented reality", Fundamentals of Wearable Computers and Augmented Reality, p. 227, 2015.

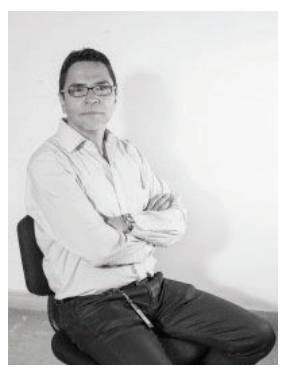

Oscar Andrés Vivas Albán. Ingeniero Electrónico de la Universidad del Cauca, Popayán, Colombia (1989). Maestría en Automática de la Universidad de Nantes, Francia (2001). Doctorado en Robótica de la Universidad de Montpellier II, Montpellier, Francia (2004). Sus líneas de interés son el control de robots y la robótica médica. Actualmente es profesor titular del Departamento de Electrónica, Instrumentación y Control de la Facultad de Ingeniería Electrónica y Telecomunicaciones de la Universidad del Cauca, en Popayán. Es el director del Grupo de Automática Industrial de la misma Universidad, escalafonado como B por Colciencias. Tiene cinco publicaciones en revistas internacionales indexadas y once ponencias en eventos internacionales. ORCID: https://orcid.org/0000-0001-5381-8234.

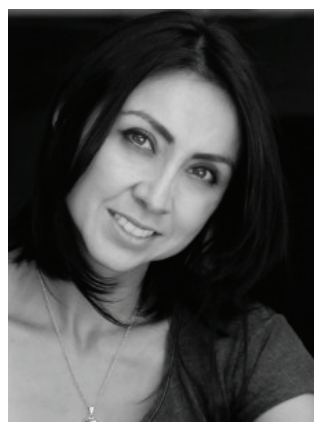

Eliana Margarita Aguilar Larrarte. Ingeniera Física de la Universidad del Cauca (2004). Magister en Ingeniería, Área Electrónica y Telecomunicaciones de la misma Universidad (2010). Sus líneas de interés son la robótica y el modelado de sistemas dinámicos. Candidata a doctor del programa de doctorado de Ciencias de la Electrónica de la Universidad del Cauca. ORCID: https://orcid.org/0000-0002-38105591.

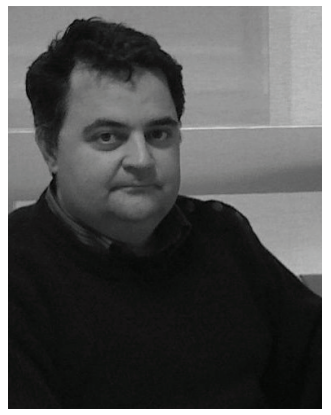

José María Sabater Navarro. Ingeniero Industrial con énfasis en Física Nuclear, Universidad Politécnica de Valencia, España, 1998. Magister en Ingeniería Mecánica de la misma universidad. Doctorado en Robótica de la Universidad Miguel Hernández de Elche, España, 2003. Desde 2003 a la fecha es profesor asociado al Departamento de Ingeniería de Sistemas y Automática de la Universidad Miguel Hernández de Elche. $\mathrm{Ha}$ realizado múltiples publicaciones y cuenta con más de siete patentes. ORCID: http://orcid.org/0000-0002-3890-6225. 